\title{
Reflujo vesicoureteral en pacientes lesionados medulares. Analisis estadístico de resultados en el tratamiento
}

\author{
Ponce Díaz-Reixa J, Sánchez Rodríguez-Losada J, Álvarez Castelo L, Romero Selas E, \\ Fernández Rosado E, González Martín M.
}

Servicio de Urología. Hospital Juan Canalejo. La Coruña.

Actas Urol Esp. 2007;31(4):366-371

\section{RESUMEN}

REFLUJO VESICOURETERAL EN PACIENTES LESIONADOS MEDULARES. ANALISIS ESTADÍSTICO DE RESULTADOS EN EL TRATAMIENTO

Introducción: El RVU en pacientes con lesión medular es una causa importante de morbilidad y mortalidad. El objetivo de este trabajo, es realizar un estudio retrospectivo, descriptivo y estadístico que analiza los resultados en el tratamiento del reflujo vesicoureteral, en los pacientes con vejiga neurógena establecida.

Material y método: Se estudian 40 pacientes con RVU, secundario a vejiga neurógena por lesión medular, entre marzo/90 y noviembre/04. Se observa RVU en el 66,2\% de las UR. La edad media es 43,05a (9-76). El 77,5\% son hombres (3,4:1). La evolución desde la lesión medular hasta el diagnóstico del RVU de 24,7 meses (0,2-87m). El tiempo de seguimiento es de 8,23a (0,529). Etiología traumática en el $70 \%$. La capacidad vesical media es de $244,9 \mathrm{ml}(43-555 \mathrm{ml})$, la compliance vesical media es de $16,12 \mathrm{ml} / \mathrm{cm} . \mathrm{H}_{2} \mathrm{O}(0,3-61,6)$. La hiperactividad del detrusor se aprecia en $72,2 \%$ y la disinergia vesico-esfinteriana externa en $71,8 \%$. El tratamiento inicial es conservador con sondaje vesical y terapia anticolinérgica.

Resultados: Curación completa del RVU en el $57,5 \%$, se reduce un $23,7 \%$ (66,2\% frente 42,5\%, p<0,02); predominantes en reflujos unilaterales. Mejoría parcial, con reducción del grado previo en 3,8\%. Progresión a grados superiores en 12,5\%. Recidivas post-curación completa en 10,9\% Los pacientes más jóvenes curan mejor (39,4 frente a 47,6 años, p=0,04). Al analizar las variables urodinámicas, no encontramos diferencias estadísticas. El tratamiento endoscópico consigue una curación del 56.3\% $(\mathrm{p}=0,18)$.

Conclusiones: Con el manejo conservador inicial, se consigue una reducción del reflujo vesicoureteral en el 23,7\%, preferentemente en aquellos pacientes con reflujo unilateral, jóvenes $(\mathrm{p}=0,04)$ y varones. La presencia o ausencia de hiperactividad o disinergia, no empeora o mejora los resultados, sin embargo se asocia con recidivas. A mayor tiempo de evolución, mejores tasas de curación, pero también mayores recidivas $(\mathrm{p}=0,007)$. El tratamiento endoscópico con sustancias abultantes, obtiene una buena tasa de curación $(56,3 \%)$, incluso en pacientes recidivados después del tratamiento conservador.

Palabras clave: Reflujo vesicoureteral. Vejiga neurógena. Tratamiento conservador.

\section{ABSTRACT}

VESICOURETERAL REFLUX IN SPINAL CORD INJURED PATIENTS. TREATMENT RESULTS AND STATISTICAL ANALYSIS Introduction: VUR in spinal cord injured patients is cause of important morbidity and mortality. The aim of this paper is to make a statistical, retrospective and descriptive study to analyze VUR treatment results, in neurogenic bladder SCI patients.

Material and method: We study 40 patients (80 renal units) with VUR in neurogenic bladders and SCI, between March, 1990 and November, 2004. Median age is 43.05 y (9-76). $77.5 \%$ of patients are males (3.4:1). Time from injury to VUR is $24.7 \mathrm{~m}(0.2-87)$. Median follow up is 8.23a (0.5-29). Traumatic lesions are most frequent (70\%). Median bladder capacity is $244.9 \mathrm{ml}(43-555)$ and median bladder compliance is $16.12 \mathrm{ml} / \mathrm{cm} \mathrm{H}_{2} \mathrm{O}(0.3-61.6)$. Detrusor overactivity is found in $72.2 \%$ and detrusor-sphincter dyssynergia in $71.8 \%$. Initial conservative treatment is done with indwelling catheter and anticholinergics

Results: Complete remission was found in $57.5 \%$ of RU, descending VUR a $23.7 \%$ (66.2\% previously to $42.5 \%$ after; p<0.02), and predominant in unilateral reflux. Partial response was found in $3.8 \%$, progression in $12.5 \%$ and recidiva post $\mathrm{CR}$ in $10.9 \%$. Younger patients have better remission rates (39.4 to $47.6 \mathrm{y}$; $\mathrm{p}=0.04)$. Urodinamycs variables don't found any significant differences in treatment results. Endoscopic treatment with bulking agents gets a good response rate $(56.3 \%)(\mathrm{p}=0.18)$.

Conclusions: Initial conservative treatment gets a $23.7 \%$ reduction of VUR, especially in men, young and unilateral reflux patients. Anyhow, we observe some progression and recidiva. Detrusor overactivity and detrusor-sphincter dyssynergia didn't influence in treatment results, but they are found in all patients with recidiva. With longer reflux evolution, we observe better responses, but also a higher recidiva rate $(\mathrm{p}=0.007)$. Endoscopic bulky injection techniques found out a good response rate (56.3\%), also in patients with recidiva after conservative treatment.

Keywords: Vesicoureteral reflux. Neurogenic bladder. Conservative treatment. 
$\mathrm{E}^{\prime}$ reflujo vesicoureteral (RVU) en pacientes con lesión medular es una causa importante de morbilidad y mortalidad. Tiene una incidencia superior (13-23\%) a la población sana adulta $(5 \%)^{1,2}$. Estos pacientes están a riesgo de presentar deterioro de la función renal, debido a la presencia de infecciones recurrentes, RVU, elevadas presiones intravesicales y anormalidades en el uréter $^{3}$. El RVU, sin un tratamiento adecuado, puede provocar hidronefrosis e infecciones urinarias ascendentes, con el consiguiente deterioro renal progresivo y fallo renal terminal ${ }^{4}$.

Los cambios que se producen en el tracto urinario inferior a consecuencia de la lesión medular, varían en el tiempo y estos cambios pueden ser evaluados urodinamicamente. La presencia de hiperactividad del detrusor, con o sin disinergia vesicoesfinteriana asociada, se demuestra como un factor de riesgo para la presencia de deterioro del tracto urinario superior (TUS). Los avances en el manejo multidisciplinar de los pacientes con lesión medular, han conseguido reducir las tasas de mortalidad específica, que anteriormente era principalmente secundaria a trastornos genitourinarios ${ }^{5}$ y que en la actualidad, son de indole cardiovascular (tabaco, cardiopatía isquémica e hipertensión) y endocrina (diabetes mellitus) $^{6}$.

Según las pautas clínicas de la EAU, el primer objetivo de tratamiento en la vejiga neurogénica, consiste en la protección del tracto urinario superior (TUS), a la vez que conseguimos la continencia del paciente, una calidad de vida aceptable y la restauración parcial o total de la función del tracto urinario inferior. El manejo del reflujo vesicoureteral en lesionados medulares, es controvertido, no existiendo en la actualidad unas pautas definidas para su manejo ${ }^{7}$.

El objetivo de este trabajo, es realizar un estudio retrospectivo, descriptivo y estadístico, que nos permite analizar los resultados en el tratamiento del reflujo vesicoureteral, en los pacientes con lesión medular y con el diagnóstico de vejiga neurógena establecida.

\section{MATERIAL Y MÉTODO}

Se realiza una búsqueda de pacientes lesionados medulares, a los que se les realizó una cistografía isotópica, para valorar la presencia de
RVU. Entre Marzo de 1990 y Noviembre de 2004 se dispone de 40 pacientes con el diagnóstico de reflujo vesicoureteral, secundario a vejiga neurógena por lesión medular.

Las variables descriptivas analizadas son edad, sexo, etiología, nivel de lesión, tiempo de evolución (desde la lesión medular hasta el diagnóstico del reflujo), tiempo de seguimiento, clínica neurológica, grado y lado del reflujo y el tipo de tratamiento utilizado. El estudio urodinámico aporta las variables de capacidad vesical, compliance vesical, volumen primera contracción no inhibida, presión de fuga, hiperactividad vesical y disinergia vésico esfinteriana. Se analizan las unidades renales de manera independiente (80 UR).

La media (rango) edad es 43,05 años (9-76). El $77,5 \%$ son hombres $(3,4: 1)$. El tiempo medio de evolución desde la lesión medular hasta el diagnóstico del RVU es de 24,7 meses $(0,2-87 \mathrm{~m})$. El tiempo de seguimiento es de 8,23 años (0,5-29). La etiología es traumática en el 70\% (28p). Las metametaras dorsales y cervicales son las más frecuentemente afectadas ( $40 \%$ y $22,5 \%$ respectivamente), lumbares $(17,5 \%)$ y sacras $(12,5 \%)$. El $20 \%$ de los pacientes, presenta tetraplejia y el $80 \%$ restante, conserva la movilidad del tren superior $(57,5 \%$ Paraplejía y 22,5\% Normal). De las 80 UR, se objetiva RVU en el 66,2\% (53 UR, 40 pac); unilateral en el $67,5 \%$ (derecho (35\%) e izquierdo (32,5\%)) y bilateral en el $32,5 \%$ (Tabla 1 y Tabla 2 ).

En el estudio urodinámico, la capacidad vesical media es de $244,9 \mathrm{ml}(43-555 \mathrm{ml})$, la compliance vesical media es de $16,12 \mathrm{ml} / \mathrm{cm} \mathrm{H}_{2} \mathrm{O}$ (0,3-61,6). Las contracciones no inhibidas aparecen a volúmenes bajos, con una media de $131 \mathrm{ml}$ (40-316). La presión media de fuga es de 73,5 $\mathrm{cmH}_{2} \mathrm{O}$ (5-292). La hiperactividad vesical se aprecia en un $72,2 \%$ y la disinergia vésico-esfinteriana externa en un $71,8 \%$ (Tabla 3 ).

Para realizar el estudio estadístico, se dispone del software estadístico informático SPSS 12.0 para Windows.

\section{RESULTADOS}

Los 40 pacientes fueron tratados de forma conservadora inicialmente y con este tratamiento, se ha conseguido la curación completa del reflujo en el 57,5\% de los casos (46 UR). Reduciéndose la incidencia de RVU en un $23,7 \%$ 
Tabla 1

\begin{tabular}{|c|c|c|c|c|}
\hline & & $\mathbf{n}$ & Media & Rango \\
\hline Edad & & 40 & 43,05 & 9-76 \\
\hline T. Evolución & & & $24,7 \mathrm{~m}$ & $0,2-87 \mathrm{~m}$ \\
\hline \multirow[t]{2}{*}{ T. Seguimiento } & & & $8,23 \mathrm{a}$ & $0,5-29 a$ \\
\hline & & $\mathbf{n}$ & $\%$ & \\
\hline \multirow[t]{2}{*}{ Sexo } & Hombre & 31 & 77,5 & \\
\hline & Mujer & 9 & 22,5 & \\
\hline \multirow[t]{6}{*}{ Etiologia } & Traumática & 28 & 70,0 & \\
\hline & Infecciosa & 1 & 2,5 & \\
\hline & Degenerativa & 1 & 2,5 & \\
\hline & Posquirúrgica & 4 & 10,0 & \\
\hline & Congénita & 4 & 10,0 & \\
\hline & Desconocida & 2 & 5,0 & \\
\hline \multirow[t]{5}{*}{ Nivel } & Cervical & 9 & 22,5 & \\
\hline & Dorsal & 16 & 40,0 & \\
\hline & Lumbar & 7 & 17,5 & \\
\hline & Sacro & 5 & 12,5 & \\
\hline & Desconocida & 3 & 7,5 & \\
\hline \multirow[t]{3}{*}{ Neurología } & Normal & 9 & 22,5 & \\
\hline & Paraplegia & 23 & 57,5 & \\
\hline & Tetraplegia & 8 & 20,0 & \\
\hline
\end{tabular}

Tabla 2

\begin{tabular}{lcc}
\hline Reflujo vesicoureteral & n & \% \\
\hline Derecho & 14 & 35 \\
No RVU & 13 & 32,5 \\
Grado I & 3 & 7,5 \\
Grado II & 8 & 20,0 \\
Grado III & 9 & 22,5 \\
Grado IV & 7 & 17,5 \\
Izquierdo & 13 & 32,5 \\
No RVU & 14 & 35,0 \\
Grado I & 6 & 15,0 \\
Grado II & 3 & 7,5 \\
Grado III & 11 & 27,5 \\
Grado IV & 6 & 15,0 \\
Bilateral & 13 & 32,50 \\
\hline
\end{tabular}

(66,2\% frente $42,5 \%, \mathrm{p}<0,02)$. En algunos casos, se consigue una mejoría parcial, con reducción del grado previo $(3,8 \%)$. Se observa, sin embargo que el $12,5 \%$ de las UR tratadas, ha progresado en este tiempo, empeorando a grados superiores. Posteriormente, 5 UR $(10,9 \%)$ han recidivado, una vez que había desaparecido el reflujo.
Las curaciones completas son predominantes en reflujos unilaterales. Los RVU derecho descendieron de un $35 \%$ a un $12,5 \%$ (RR 1,55 (IC 95\% 1,05-2,23); $\mathrm{p}<0,03)$ y los del lado izquierdo desde un $32,5 \%$ a un $17,5 \%$ (RR 2,16 (IC $95 \%$ 1,20-3,9); $\mathrm{p}<0,0001)$ Sin embargo, los reflujos bilaterales, han permanecido casi iguales $(32,5 \%$ frente a un $30 \%$ ). Los reflujos que no curaron con el tratamiento conservador, son más frecuentemente moderado-severos; grado III $(18,8 \%)$ y grado IV (10\%).

Para valorar que variables puedan influir en las tasas de curación y encontrar factores pronósticos, se realizó un estudio estadístico comparando las tasas de curación en las diferentes variables estudiadas. Los pacientes más jóvenes curan mejor (39,4 frente a 47,6 años, p=0,04). En la variable sexo, no encontramos diferencias significativas $(\mathrm{p}=0,09)$, pero vemos como el $61,3 \%$ de los varones se curan, frente a solo el $38,9 \%$ de las mujeres. En las variables etiología, clínica neurológica y nivel de la lesión, tampoco encontramos diferencias estadísticas.

Al analizar las variables urodinámicas, no encontramos diferencias estadísticas con la capacidad vesical $(p=0,173)$, compliance $(p=0,355)$, volumen primera contracción $(\mathrm{p}=0,613)$, hiperactividad detrusor $(\mathrm{p}=0,516)$ y disinergia vesicoesfinteriana $(\mathrm{p}=0,513)$.

Los pacientes que presentan recidiva $(10,9 \%)$, están asociados con lesiones traumáticas o posquirúrgicas y a nivel dorsal. Estos presentan datos urodinámicos de riesgo, con hiperactividad del detrusor y disinergia vesicoesfinteriana (todas estas sin significación estadística) y un mayor tiempo de enfermedad $(36,3 \mathrm{~m}$ frente a $20,7 \mathrm{~m}$, $\mathrm{p}=0,007$ ).

El tratamiento endoscópico fue realizado en 32 UR (40\%), consiguiendo una tasa de curación del 56,3\% (18UR) de las UR tratadas (p=0.18). En 6 UR $(18,7 \%)$ fue necesaria una segunda inyección de Macroplastique. A 4 pacientes (10\%, 8UR) se les colocó una prótesis endouretral, desapareciendo el RVU en el 87,5\% (7UR). En los casos de ampliación vesical (5 pac, 10UR), el RVU solo desapareció en la mitad de las UR. Los casos de recidiva (5 UR), se trataron con inyección endoscópica, desapareciendo el RVU en todas ellas (Tabla 4). 
Tabla 3

Estudio urodinámico de los pacientes estudiados

\begin{tabular}{lcccc}
\hline Urodinámica & Media & D. standard & Mediana & Rango \\
\hline Capacidad Vesical (ml) & 244,93 & 120,59 & 268 & $53-555$ \\
Compliance (ml/cmH $\left.{ }_{2} \mathrm{O}\right)$ ) & 16,12 & 15,08 & 10,9 & $0,3-61,6$ \\
Volumen 1 a CNI (ml) & 131 & 83,27 & 130 & $40-316$ \\
Presión de Fuga $\left(\mathrm{cmH}_{2}\right.$ O) & 73,5 & 92,45 & 52 & $5-292$ \\
\hline & $\mathbf{n}$ & $\%$ & \\
\hline Disinergia DE & 26 & $72,2 \%$ & & \\
Hiperactividad & 28 & $71,8 \%$ & & \\
\hline
\end{tabular}

Tabla 4

Estudio estadístico de las diferentes variables según curación con tratamiento conservador

\begin{tabular}{|c|c|c|c|c|}
\hline & & Curación & No curación & Valor de $\mathbf{p}$ \\
\hline \multicolumn{2}{|l|}{ Edad (años) } & 39,4 & 47,6 & 0,04 \\
\hline \multicolumn{5}{|l|}{ Sexo } \\
\hline & Varón & $61,3 \%$ & $38,7 \%$ & \multirow[t]{2}{*}{0,08} \\
\hline & Mujer & $38,9 \%$ & $61,1 \%$ & \\
\hline Tiempo Evolución (meses) & & 114,2 & 97,45 & 0,53 \\
\hline \multicolumn{2}{|l|}{ Etiologia } & - & - & n.s \\
\hline \multicolumn{2}{|l|}{ Nivel lesion } & - & - & n.s \\
\hline \multicolumn{2}{|l|}{ Capacidad vesical (ml) } & 265,3 & 227,13 & 0,17 \\
\hline \multicolumn{2}{|l|}{ Compliance vesical (ml/cm H2O) } & 19,4 & 13,2 & 0,35 \\
\hline \multicolumn{2}{|l|}{ Vol $1^{a} \mathrm{a} C N$} & 135,3 & 127,7 & 0,61 \\
\hline \multirow[t]{2}{*}{ Hiperactividad Detrusor } & $\mathrm{Si}$ & $57,1 \%$ & $42,9 \%$ & \multirow[t]{2}{*}{0,51} \\
\hline & No & $54,5 \%$ & $45,5 \%$ & \\
\hline \multirow[t]{2}{*}{ Disinergia VE } & $\mathrm{Si}$ & $51,9 \%$ & $48,1 \%$ & \multirow[t]{2}{*}{0,51} \\
\hline & No & $55,0 \%$ & $45,0 \%$ & \\
\hline
\end{tabular}

\section{DISCUSIÓN}

En este estudio, se valora la curación del reflujo después del tratamiento inicial, conservador (curación completa, parcial o progresión) y se realiza un estudio estadístico, para valorar la significación estadística entre las variables estudiadas y la posibilidad de curación con el tratamiento conservador. Asimismo, se analizan las tasas de curación que se consigue con otros tipos de tratamientos endoscópicos (inyección de sustancias abultantes o prótesis endouretral) o quirúrgicos (ampliación vesical).

El objetivo principal en el manejo urológico en los pacientes lesionados medulares es asegurar un vaciado vesical adecuado, con un llenado y un vaciado a baja presión. Esto permite prevenir las infecciones urinarias, el daño de la pared vesical, la sobredistensión vesical, el reflujo vesicoureteral y la litiasis.

La disfunción neurogénica de la vejiga, en los pacientes lesionados medulares, puede conllevar a elevadas presiones intravesicales e infecciones urinarias de repetición. Esto, sumado a la presencia de reflujo vesicoureteral, puede desencadenar un deterioro progresivo del tracto urinario superior y pérdida de la función renal.

El manejo en nuestra unidad, es inicialmente conservador, persiguiendo unas condiciones vesicales de baja presión, mejor compliance y bajas presiones de vaciado vesical. Para esto, los pacientes realizan cateterismos intermitentes o llevan una sonda vesical permanente. Asimismo, se añade terapia anticolinérgica para controlar la hiperactividad vesical en los casos en que la prueba urodinámica lo demuestra. Con este tratamiento, obtenemos un porcentaje de curación del $57,5 \%$ de las unidades renales tratadas, similar a otra serie publicada $^{7}$. El tratamiento conservador necesita de un seguimiento clínico estrecho, para detectar empeoramiento o recidivas del reflujo. En nuestra serie, observamos un empeoramiento del 12,5\% de las unidades renales y un $10,9 \%$ de recidivas. Es importante mantener una terapia conservadora, una vez conseguida la curación completa, para evitar las recidivas.

Foley $\mathrm{SJ}^{7}$ et al. hacen una revisión de los pacientes vistos en un centro de referencia para lesionados medulares. Observan que el $8 \%$ de los pacientes lesionados medulares, tienen algún grado de RVU. El tratamiento inicial es conservador, mediante cateterización intermitente y terapia anticolinérgica. Con este tratamiento, consiguen la curación completa en el 53\%, mejora par- 
cialmente en el $11 \%$ y permanece en el $36 \%$. En los casos, en los que no mejora el RVU, se realizó inyección de sustancias abultantes, con una tasa de curación de 60\% (4 pacientes de 6 tratados). Aconsejan tratar a los pacientes con reflujo y elevadas presiones vesicales, tan pronto se diagnostique el reflujo, pero prefieren terapia conservadora en vejigas de baja presión, no infección y asintomáticos. En los casos en los que la terapia conservadora no es eficaz, consideran una buena opción la terapia endoscópica de sustancias abultantes.

Weld KJ et al.. en un estudio retrospectivo, revisan la evolución de la compliance vesical con el tiempo, en función del tipo de manejo utilizado. La conclusión es que una baja compliance, se asocia a reflujo vesicoureteral $(\mathrm{p}<0,01)$, anormalidades del tracto urinario superior, pielonefritis y litiasis vesical. Los cateterismos intermitentes protegen la compliance vesical, independientemente del nivel y grado de la lesión medular ${ }^{8}$.

Giannantoni $A$ et al evalúan la incidencia de complicaciones del TUS en pacientes con cateterismos intermitentes y maniobras de estimulación suprapúbica, relacionándolo con la clínica y los datos urodinámicos. Observan un $22 \%$ de complicaciones del TUS, con una incidencia significativamente menor, de hidronefrosis $(\mathrm{p}=0,03)$ y RVU $(p=0,04)$ en el grupo de los cateterismos intermitentes. Concluyen también que los pacientes con complicaciones del TUS, cateterización intermitente y elevadas presiones, pueden ser subsidiarios de tratamiento anticolinérgico ${ }^{9}$.

En los casos en los que no se consigue una curación completa y el paciente presenta datos urodinámicos de hiperactividad o disinergia vesicoesfinteriana, se plantea la posibilidad de realizar un tratamiento endoscópico. Las inyección de sustancias abultantes en el meato ureteral, inicialmente Teflon y posteriormente Macroplastique, suponen un buen método para tratar el reflujo, con una baja morbilidad y mortalidad. En nuestra serie, obtenemos una tasa de curación del $56,3 \%$ de las unidades renales tratadas.

Pelletier $A K$ et al. ${ }^{10}$ hacen una revisión de los resultados en el tratamiento endoscópico del RVU, con colágeno, polytetrafluoroetileno (Teflon $\odot$ ) y polydimetilsiloxano (Macroplastique $\odot$ ). Se observan unas tasas de curación altas con Teflón
(80\%) y Macroplastique (86\%); sin embargo, el colágeno no se aprecia como un tratamiento eficaz.

\section{CONCLUSIONES}

Los pacientes lesionados medulares, que padecen RVU, son mayoritariamente varones de edad media, con lesiones traumáticas. Conlleva un periodo largo de convalecencia, con gran riesgo de presentar complicaciones del tracto urinario y consiguiente deterioro de la función renal. El RVU se presenta años después de la lesión medular, luego se precisan controles urológicos para detectarlo y corregirlo. En las pruebas urodinámicas se aprecian vejigas con compliance y capacidad vesical reducida y porcentajes elevados de disinergia vesicoesfinteriana e hiperactividad del detrusor.

Con el manejo conservador inicial, se consigue una reducción del reflujo vesicoureteral en el $23,7 \%$, preferentemente en aquellos pacientes con reflujo unilateral. En nuestra serie, los pacientes varones curan mejor $(61,3 \%)$ que las mujeres. Los pacientes de menor edad tienen también mejores resultados $(p=0,04)$. En el estudio urodinámico, hemos observado que una mejor capacidad y compliance vesical, obtienen mejores tasas de curación, pero sin llegar a observar diferencias significativas. Pero la presencia de hiperactividad del detrusor y disinergia vesicoesfinteriana, no empeora los resultados. Sin embargo en los pacientes que recidivan (10,9\%), observamos en todos ellos hiperactividad y disinergia, sin significación estadística. A mayor tiempo de evolución, se obtienen mejores tasas de curación, pero también mayores recidivas $(\mathrm{p}=0,007)$.

El tratamiento endoscópico con sustancias abultantes, obtiene una buena tasa de curación $(56,3 \%)$, siendo necesaria una segunda inyección en el $18,7 \%$. Las recidivas del tratamiento conservador pueden ser tratadas eficazmente con sustancias abultantes.

\section{REFERENCIAS}

1. Bors E, Comarr AE. Vesicoureteric reflux in paraplegic patients. J Urol. 1952;68(4):691-698.

2. Estes RC, Brooks RT. Vesicoureteral reflux in adults. J Urol. 1970;103(5):603-605. 
3. Hutch JA. Vesico-ureteral reflux in paraplegic: cause and correction. J Urol. 1952;68(2):457-469.

4. Talbot HS, Bunts RC. Late renal changes in paraplejía: Hydronephrosis due to vesico-ureteral reflux. J Urol. 1949; 61:810.

5. Hackler RH. A 25-year prospective mortality in the spinal cord injured patient: Comparison with the long-term living paraplegic. J Urol 1977;117(4):486-492.

6. Garschick E, Kelley A, Cohen SA, Garrison A, Tun CG, Gagnon D, Brown R. Spinal Cord. 2005;43(7):408-416.

7. Foley SJ, McFarlane JP, Shah PJ. Vesico-ureteric reflux in adult patients with spinal injury. Br J Urol. 1997;79(6):888 891.

8. Weld KJ, Graney MJ, Dmochowski RR. Differences in bladder compliance with time and associations of bladder management with compliance in spinal cord injured patients. J Urol. 2000;163(4):1228-1233.
9. Giannantoni A, Scivoletto G, Di Stasi SM, Silecchia A, Finazzi-Agro E, Micali I, et al. Clean intermittent catheterization and prevention of renal disease in spinal cord injury patients. Spinal Cord. 1998;36(1):29-32.

10. Pelletier AK, Anderson PA, Schwarz RD. Comparison of different substances for subureteric injection in the management of vesicoureteric reflux in children. Can $J$ Urol. 2005; 12(4):2774-2777.

Correspondencia autor: Dr. J. Ponce Díaz-Reixa Servicio de Urología. Hospital Juan Canalejo Xubias de Arriba, 84. 15006 La Coruña.

Tel.: 981178000

E-mail autor: ponce@canalejo.org

Información artículo: Original - Vejiga neurógena

Trabajo recibido: enero 2007

Trabajo aceptado: febrero 2007 